\title{
Immune complex nephritis in alcoholic cirrhosis: detection of Mallory body antigen in complexes by means of monoclonal antibodies to Mallory bodies
}

\author{
J BURNS, AJ D'ARDENNE, JA MORTON, J O’ D McGEE \\ From the University of Oxford, Nuffield Department of Pathology John Radcliffe Hospital, Oxford OX2 \\ $9 D U$
}

SUMMARY A Mallory body (alcoholic hyaline) antigen (JMB2) which is also present in intermediate filaments of epithelial origin was demonstrated immunohistochemically in renal glomeruli of three out of eleven patients with alcoholic liver damage. In two of these patients, both of whom had alcoholic cirrhosis with Mallory bodies, it was associated with mesangial deposits of $\operatorname{IgA}$ and C3. JMB2 was not found in glomeruli of normal controls, nor in a series of cases of glomerulonephritis in non-alcoholic patients. It is concluded that JMB2 is present in immune complexes in renal glomeruli of patients with renal disease consequent on alcoholic liver disease.

Glomerular abnormalities, in particular mesangial expansion, are frequently found in patients with alcoholic liver disease and these are associated with mesangial deposits of IgA. ${ }^{2}$ They are sometimes but not always associated with clinical manifestations of glomerulonephritis and renal failure. ${ }^{2-4}$ The aetiology of these changes is not established but they are often accompanied by high concentrations of $\operatorname{IgA}$ in serum. ${ }^{5}$ The latter is present in both monomeric and polymeric forms, and in immune complexes. ${ }^{67}$ It has been suggested that patients with cirrhosis develop circulating immune complexes as a result of defective hepatic sequestration of gut associated antigens. ${ }^{8}$ Alternatively, it has been speculated that these complexes may be derived from IgA aggregates combining with liver cell membrane proteins or with Mallory body (MBs) proteins from alcohol damaged liver.'

In this paper two monoclonal antibodies (antiJMB1 and anti-JMB2) directed against MBs/ intermediate filaments were used to determine whether MB-related antigens are present in the glomeruli of patients with alcoholic liver disease.

It is demonstrated by immunohistochemistry that JMB2 is present in glomerular mesangium and capillaries in some cases together with $\operatorname{IgA}$ and $\mathrm{C} 3$ in patients with alcoholic cirrhosis.

Accepted for publication 15 February 1983

\section{Material and methods}

Samples of liver and kidney were obtained from 11 cases of alcoholic liver disease at postmortem, and from six controls. The controls consisted of one patient with primary biliary cirrhosis, one with a history of excessive alcohol intake but no liver pathology, and four patients without evidence of either hepatic or renal disease. Renal biopsies were obtained from patients with Berger's IgA nephropathy (1), segmental glomerulonephritis (1), membranoproliferative glomerulonephritis (4), membranous glomerulonephritis (5), crescentic glomerulonephritis (2); four morphologically normal renal biopsies were also included in the series. Part of the tissue from each case was processed for routine histology and the remainder for immunohistochemistry. Immunoglobulins and complement components were detected by direct immunofluorescence, and MB antigens by indirect immunoperoxidase. ${ }^{10}$ All immunohistochemistry was performed on cryostat sections of frozen tissue. Two Mallory body derived monoclonal antibodies were used in the form of culture supernatant. These were anti-JMB1, which reacts preferentially with Mallory bodies," and anti-JMB2 which reacts with Mallory bodies and epithelial intermediate filaments. ${ }^{1}$ The preparation and immunohistochemical staining characteristics of these antibodies have been documented previously. ${ }^{1011}$ 
Table 1 Hepatic and glomerular pathology in patients with alcoholic liver disease

\begin{tabular}{|c|c|c|c|c|c|c|c|c|}
\hline \multirow[t]{2}{*}{ Case No } & \multicolumn{2}{|c|}{ Liver pathology } & \multicolumn{6}{|c|}{ Glomerular pathology* } \\
\hline & Diagnosis & Mallory bodies & $\operatorname{Ig} A$ & $\operatorname{Ig} G$ & $\operatorname{Ig} M$ & C3 & $C l q$ & $J M B 2$ \\
\hline $\begin{array}{r}1 \\
2 \\
3 \\
4 \\
5 \\
6 \\
7 \\
8 \\
9 \\
10 \\
11\end{array}$ & $\begin{array}{l}\text { AC } \\
\text { AC } \\
\text { AC } \\
\text { AC } \\
\text { AC } \\
\text { AC } \\
\text { AC } \\
\text { AC } \\
\text { AH } \\
\text { AH } \\
\text { AH }\end{array}$ & $\begin{array}{l}++++ \\
+ \\
+ \\
0 \\
++ \\
+ \\
0 \\
+++ \\
+++ \\
+++ \\
+++ \\
+++\end{array}$ & $\begin{array}{l}++ \\
++ \\
++ \\
0 \\
0 \\
0 \\
0 \\
0 \\
0 \\
0 \\
0\end{array}$ & $\begin{array}{l}+ \\
+ \\
0 \\
0 \\
0 \\
0 \\
0 \\
0 \\
0 \\
0 \\
0\end{array}$ & $\begin{array}{l}+ \\
+ \\
+ \\
+ \\
0 \\
+ \\
+ \\
0 \\
0 \\
0 \\
0\end{array}$ & $\begin{array}{l}+ \\
+ \\
0 \\
0 \\
0 \\
+ \\
+ \\
0 \\
0 \\
0 \\
0\end{array}$ & $\begin{array}{l}0 \\
0 \\
+ \\
+ \\
0 \\
+ \\
+ \\
0 \\
0 \\
0 \\
0\end{array}$ & $\begin{array}{l}++ \\
+ \\
0 \\
0 \\
0 \\
0 \\
0 \\
0 \\
+ \\
0 \\
0\end{array}$ \\
\hline
\end{tabular}

$\mathrm{AC}=$ alcoholic cirrhosis $\quad \mathrm{AH}=$ alcoholic hepatitis

* + to ++++ is an arbitrary score based on subjective assessment of the quantity of immunofluorescence or immunoperoxidase deposits.

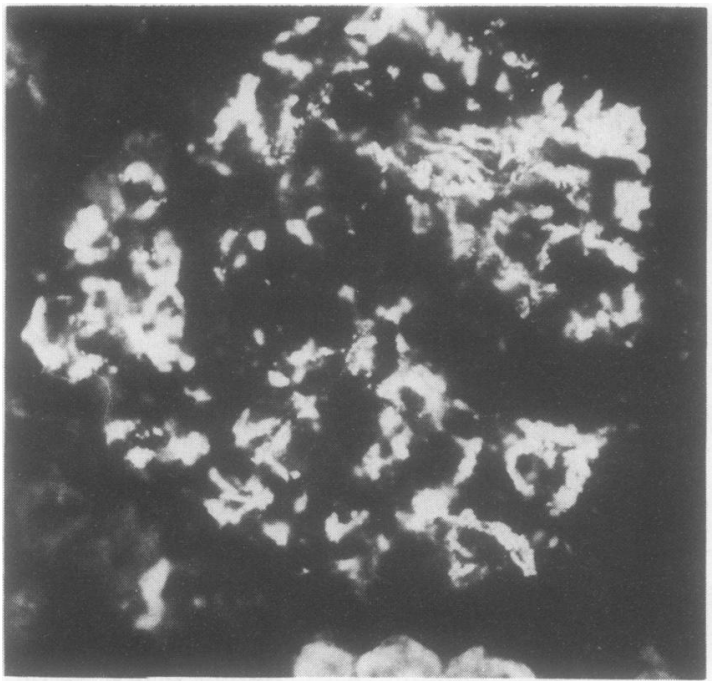

Fig. 1 IgA deposition in mesangium of patient with alcoholic cirrhosis. Immunoftuorescence $\times 350$

\section{Results}

Of the 11 cases of alcoholic liver disease, eight had cirrhosis and three had severe alcoholic hepatitis (Table 1). The majority had MBs within hepatocytes detectable on both routine light microscopy and on staining with anti-JMB1 and anti-JMB2. There was a variable degree of fatty infiltration.

By light microscopy the kidneys from patients with alcoholic liver disease showed only minor changes. These included mesangial expansion in a few cases and occasional basement membrane thickening. Three cases of alcoholic cirrhosis had extensive deposition of $\operatorname{IgA}$ in the mesangium of glomeruli (Fig. 1) accompanied by varying amounts

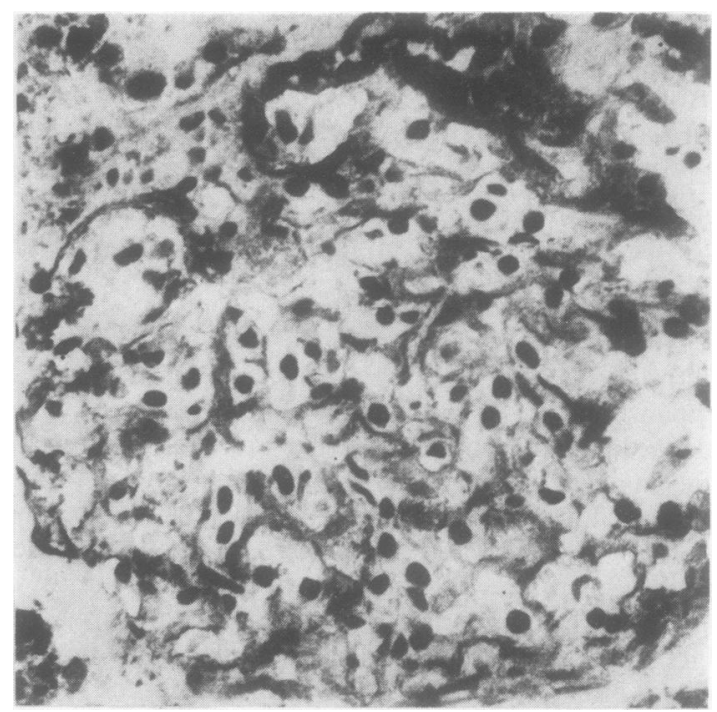

Fig. 2 JMB2 deposition in mesangium and capillary loops in patient with alcoholic cirrhosis. Immunoperoxidase $\times$ 400

of IgG, IgM, C3 and C1q (Table 1). In two of these patients mesangial and capillary JMB2 (Fig. 2) was

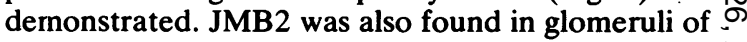
one of the three cases of alcoholic hepatitis but $\tilde{O}$ without accompanying immunoglobulin or comple- స్ ment. Two cases of alcoholic cirrhosis had small glomerular deposits of IgM, C3 and C1q without 0 evidence of either IgA or JMB2. Glomerular staining for JMB1 was negative in all patients. JMB1 and $\stackrel{\mathscr{\infty}}{\rightarrow}$ JMB2 were not detected in the glomeruli of one 0 patient with primary biliary cirrhosis, in one alcoholic without liver pathology and in four cases with normal liver and renal structure and function.

In normal kidney JMB2 was present in renal tubular epithelium and in parietal cells lining Bowman's

(1) 
Table 2 Clinical details of patients with alcoholic liver disease

\begin{tabular}{|c|c|c|c|c|c|}
\hline Case No & Diagnosis & Cause of death & $\begin{array}{l}\text { Blood urea } \\
\text { (mmolll) }\end{array}$ & $\begin{array}{l}\text { Serum IgA } \\
(g / l)\end{array}$ & Urine \\
\hline $\begin{array}{r}1 \\
2 \\
3 \\
4 \\
5 \\
6 \\
7 \\
8 \\
9 \\
10 \\
11\end{array}$ & $\begin{array}{l}\text { AC } \\
\text { AC } \\
\text { AC } \\
\text { AC } \\
\text { AC } \\
\text { AC } \\
\text { AC } \\
\text { AC } \\
\text { AH } \\
\text { AH } \\
\text { AH }\end{array}$ & $\begin{array}{l}\text { Hepatic failure } \\
\text { Bronchopneumonia and meningitis } \\
\text { Bronchopneumonia } \\
\text { Ruptured oesophageal varices } \\
\text { Hepatic failure } \\
\text { Bronchopneumonia, carcinoma of pharynx } \\
\text { Lobar pneumonia } \\
\text { Hepatic failure } \\
\text { Cerebral haemorrhage } \\
\text { Bronchopneumonia } \\
\text { Ruptured abdominal aortic aneurysm }\end{array}$ & $\begin{array}{l}9 \cdot 43 \\
\mathbf{N} \\
\mathbf{N} \\
\mathbf{N} \\
8 \cdot 0 \\
\mathbf{N} \\
\mathbf{N} \\
26 \cdot 3 \\
\mathbf{N} \\
\mathbf{N} \\
-\end{array}$ & $\begin{array}{l}\overline{17 \cdot 2} \\
= \\
= \\
\overline{-} \\
\bar{N} \\
=\end{array}$ & $\begin{array}{l}\mathbf{N} \\
\mathbf{R B C} \\
\mathbf{N} \\
\mathbf{N} \\
\mathbf{N} \\
\mathbf{N} \\
\mathbf{N} \\
\mathbf{N} \\
\mathbf{N} \\
\mathbf{N} \\
-\end{array}$ \\
\hline
\end{tabular}

$\mathbf{N}=$ normal.

$-=$ not recorded

RBC $=$ red blood cells

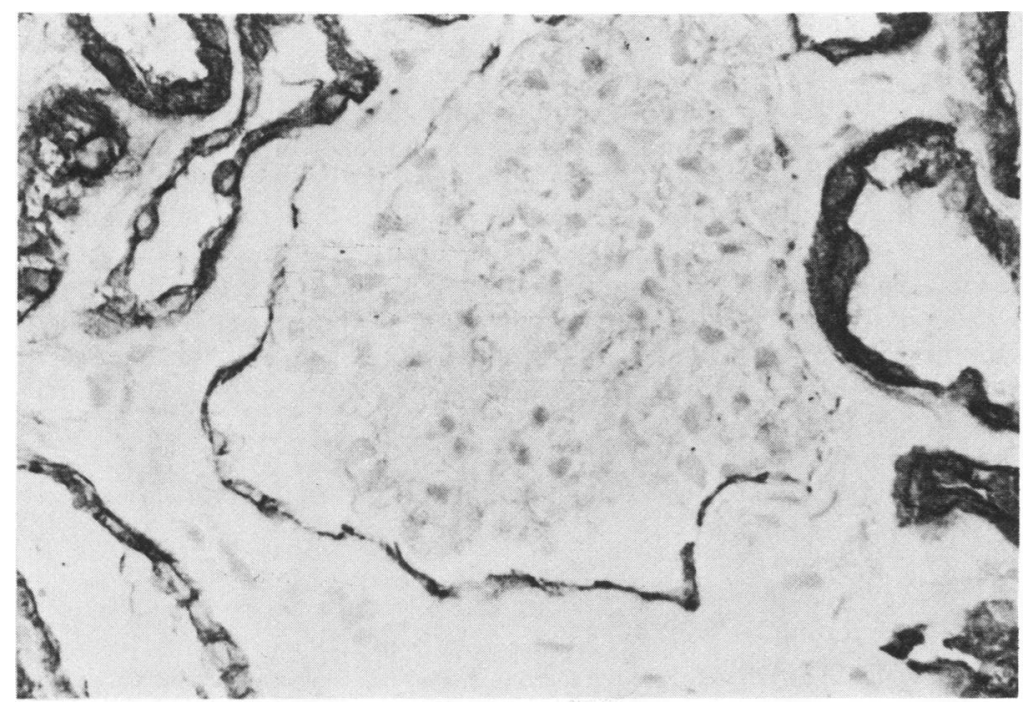

Fig. $3 \quad J M B 2$ distribution in normal kidney. This antigen is in renal proximal and distal tubules and in Bowman's capsule.

Immunoperoxidase $\times 250$

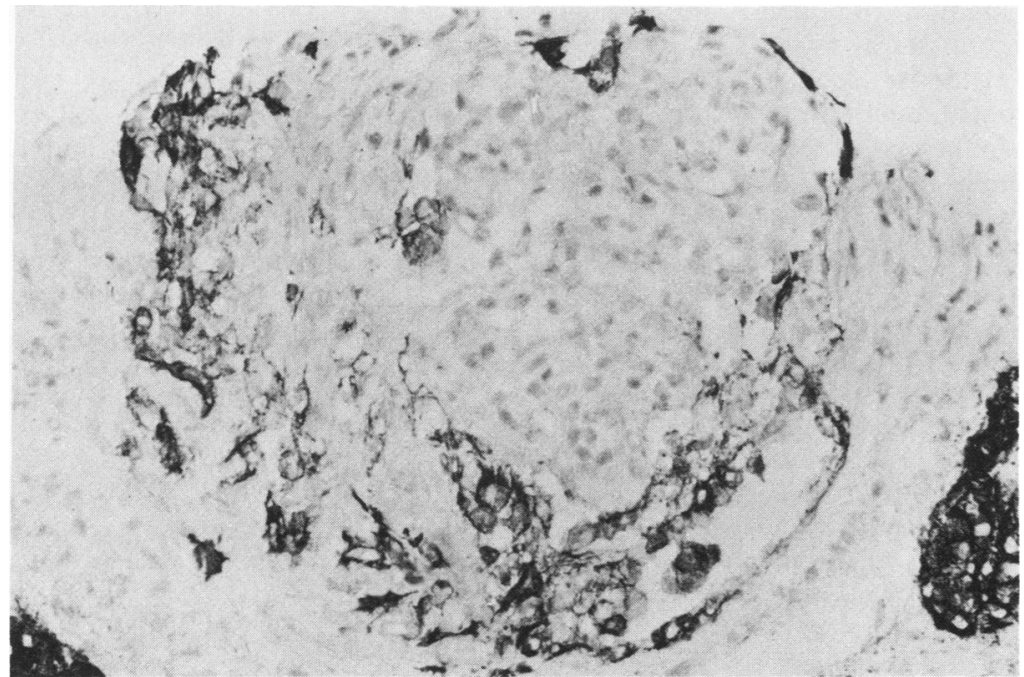

Fig. $4 J M B 2$ deposition in crescentic nephritis. JMB2 is only detectable in proliferating epithelial cells. Immunoperoxidase $\times 250$ 
capsule (Fig. 3) and JMB1 to a lesser extent. JMB1 and JMB2 were not found in glomerular tufts. In the series of cases of glomerulonephritis unrelated to liver disease, JMB2 was only detected in proliferating epithelial cells of crescentic nephritis (Fig. 4).

The principal clinical details and causes of death in the alcoholic patients are given in Table 2. Only one patient (case 2) had clinical evidence of glomerular disease in that he had microscopic haematuria. This patient also had mesangial $\operatorname{IgA}$ and JMB2. Three other cases (1, 5 and 8) had impaired renal function with raised plasma urea and creatinine, but at no time was blood or protein found in the urine. Serum $\operatorname{IgA}$ was not measured in all patients. Where it was recorded it was raised in two cases (2 and 9). Hepatitis B antigen was not detected in serum of any of the patients.

\section{Discussion}

The finding of $\operatorname{IgA}$ in glomerular mesangium of almost half the patients with alcoholic cirrhosis is in accord with previous observations, as are the findings on light microscopy and clinical investigation. ${ }^{24}$ The observation that the antigen JMB2 is sometimes present in association with this mesangial IgA raises the possibility that they are present as immune complexes. Proof of such a relation would require elution of antibody from kidney and identification of its specificity; the circumstantial evidence is nevertheless strong. The finding of JMB2 without immunoglobulin in one case of alcoholic hepatitis does not preclude its presence in immune complexes in cirrhosis, since glomerular binding of antigen may precede stimulation of an antibody response.

If JMB2 is indeed involved in immune complex production in cirrhosis, the question arises as to its origin. The most obvious source would be alcohol damaged hepatocytes. JMB2 is a constituent of Mallory bodies and these were present in the majority of cases examined. Circulating alcoholic hyaline and antibody to alcoholic hyaline have been reported previously, ${ }^{12}$ although these finding have not been confirmed. An alternative explanation is that glomerular bound JMB2 is derived from renal tubular epithelial cells, gut or pancreas ${ }^{11}$ since it is a normal constituent of the epithelial cells of these tissues. Immune complex glomerulonephritis induced by renal tubular antigen has been reported in animals ${ }^{13}$ and renal tubular antigen has been demonstrated in membranous glomerulonephritis in man. ${ }^{14}$ This antigen was derived from proximal tubular brush border and did not have the cytoplasmic distribution of JMB2. Furthermore no normal kidney nor the cases of glomerulonephritis examined, including five cases of membranous glomerulonephritis and one of Berger's $\operatorname{IgA}$ nephropathy, had detectable JMB2 in glomerular tufts.

Only one of the patients with alcoholic liver disease in this series had clinical evidence of glomerulonephritis as manifest by microscopic haematuria. This corresponds with previous observations $s^{5}$ and lends weight to the hypothesis that renal immune complex deposition per se does not necessarily give rise to clinical renal disease. Other important factors are probably "nephritogenicity" of bound antigen and host immune response. ${ }^{15}$

Three alcoholic patients had small deposits of IgM and complement but no JMB2 or IgA. Circulating antigens other than those directly related to liver damage may have been present terminally and it is noteworthy that several died with overwhelming infections.

The possibility that JMB2 is involved in the production of immune complexes in alcoholic cirrhosis may be clinically significant not only because of the potentially damaging effect of the complexes themselves, but also because it provides evidence of an autoimmune response in these patients. Such a reaction may be important in the perpetuation of liver damage initiated by alcohol and in the genesis of renal complications in patients with alcoholic liver disease.

Mrs V Macintosh typed this manuscript.

\section{References}

' Berger J, Yaneva H, Nabarra B. Glomerular changes in patients with cirrhosis of the liver. Adv Nephrol 1977;7:3-14.

${ }^{2}$ Nakamoto Y, Lida H, Kobayashi K, Dohi K, Kida H, Hattori N, Takeuchi J. Hepatic glomerulonephritis. Virchows Archiv[A] 1981;392:45-54.

${ }^{3}$ Nochy D, Callard P, Bellon B, Bariety J, Druet P. Association of overt glomerulonephritis and liver disease: a study of 34 patients. Clin Nephrol 1976;6:422-7.

4 Callard P, Feldmann G, Prandi D, et al. Immune complex type glomerulonephritis in cirrhosis of the liver. Am J Pathol 1975;80:329-40.

s Iturriaga H, Perede T, Estévez A, Ugarte G. Serum immunoglobulin A changes in alcoholic patients. Ann Clin Res 1977;9:39-43.

- Penner E, Albini B, Milgrom F. Detection of circulating immune complexes in alcoholic liver disease. Clin Exp Immunol 1978;34:28-31.

' Sancho J, Egido J, Sánchez-Crespo M, Blasco R. Detection of monomeric and polymeric IgA containing immune complexes in serum and kidney from patients with alcoholic liver disease. Clin Exp Immunol 1981;47:327-35.

${ }^{3}$ Gormley AA, Smith PS, Seymour AE, Clarkson AR, Woodroffe AJ. IgA glomerular deposits in experimental cirrhosis. Am J Pathol 1981;104:50-4.

- Swerdlow MA, Chowdhury LN, Horn T. Patterns of IgA deposition in liver tissues in alcoholic liver disease. Am J Clin Pathol 1982;77:259-66. 
${ }^{10}$ Morton JA, Bastin J, Fleming KA, McMichael A, Burns J, McGee J O'D. Mallory bodies in alcoholic liver disease: identification of cytoplasmic filament/cell membrane and unique antigenic determinants by monoclonal antibodies. Gut 1981;22:1-7.

" McGee J O'D, Morton JA, Barbatis C et al. Monoclonal antibodies to Mallory Bodies/Intermediate Filaments and HLA (Class 1) Antigen in human liver disease. McMichael A, Fabre JW, eds. Monoclonal antibodies in clinical medicine London: Academic Press, 1982:431-55.

12 Kanagasundaram N, Kakumu S, Chen T, Leevy CM. Alcoholic hyalin antigen (AHAg) and antibody (AHAb) in alcoholic hepatitis. Gastroenterology 1977;73:1368-73.

${ }^{13}$ Edgington TS, Glassock RJ, Dixon FJ. Autologous immune complex nephritis induced with renal tubular antigen. I.
Identification and Isolation of the pathogenetic antigen. $J$ Exp Med 1968;127:555-72.

${ }^{14}$ Naruse T, Kitamura K, Miyakawa Y, Shibata S. Deposition of renal tubular epithelial antigen along the glomerular capillary walls of patients with membranous glomerulonephritis. $J$ Immunol 1973;110:1163-6.

15 Williams DG. New ideas in the pathogenesis of nephritis. J Clin Pathol 1981;34:1223-7.

Requests for reprints to: Professor J O'D McGee, University of Oxford, Nuffield Department of Pathology, John Radcliffe Hospital, Oxford OX2 9DU, England. 